\title{
SATURATED FAMILIES
}

\author{
MARTIN GOLDSTERN, HAIM I. JUDAH, AND SAHARON SHELAH
}

(Communicated by Andreas R. Blass)

\begin{abstract}
We will show that $V=K$ implies that there exist saturated (or completely separable) almost disjoint families on sets of any infinite cardinality.
\end{abstract}

\section{INTRODUCTION}

Definition. For a family $\mathfrak{A} \subseteq[X]^{\omega}$, let $I_{\mathfrak{A}}$ be the following ideal: $x \in I_{\mathfrak{A}} \leftrightarrow x$ is almost covered by finitely many elements of $\mathfrak{A}$. ("almost" means "except for finitely many points").

Let $I_{\mathfrak{A}}^{+}=\mathfrak{P}(X)-I_{\mathfrak{A}}$ (= the positive sets modulo this ideal).

Definition. An almost disjoint family $\mathfrak{A} \subseteq[X]^{\omega}$ is called saturated if for all $Y \in I_{\mathfrak{A}}^{+}$there exists an $a \in \mathfrak{A} a \subseteq Y$.

Definition. Let $S(\kappa)$ abbreviate the statement "there exists an infinite almost disjoint saturated family $\mathfrak{A} \subseteq[\kappa]^{\omega}$."

[BDS] exhibit several conditions that imply that every almost disjoint family on $\omega$ can be refined to a saturated family: $C H$ (or $M A$ ) are sufficient, or even the following consequence of $M A$ : "Every maximal almost disjoint family on $\omega$ has $2^{\omega}$ many elements."

The question whether $S\left(\aleph_{2}\right)$ holds is Problem 37 in [EH1], but [EH2] mentions Baumgartner's result $C H \rightarrow \forall n S\left(\aleph_{n}\right)$.

[K] considers a related problem: Let us call an almost disjoint family $\subseteq[X]^{\omega} \omega_{1}$-saturated if it is saturated with respect to all uncountable sets, i.e. every uncountable $Y \subseteq X$ contains an element of the family. [K] shows (from $V=L$ ) that $\forall \kappa<\aleph_{\omega_{1}}$ there exists an $\omega_{1}$-saturated family on $\kappa$.

[HJS] prove that a finite support iteration of length $\omega_{1}$ of Hechler reals will yield a model in which

$$
\forall \kappa S(\kappa)
$$

Received by the editors March 20, 1989 and, in revised form, May 15, 1990.

1980 Mathematics Subject Classification (1985 Revision). Primary 03E05, 04A20.

Key words and phrases. Almost disjoint families, completely separable families, saturated families.

The third author was supported by the BSF (publication 399). 
holds, and the question is asked, whether the same is in fact true for every model for ZFC, or even for $L$. We will prove that under following assumptions (1)(3), $\forall \kappa S(\kappa)$ will hold:

(1) $S(\omega)$,

(2) $\forall \kappa: \kappa>c f(\kappa)=\omega \rightarrow \square_{\kappa}$,

(3) $\forall \kappa: \kappa>c f(\kappa)=\omega \rightarrow$

$$
\exists \mathfrak{C} \subseteq[\kappa]^{\omega}, \operatorname{card}(\mathfrak{C})=\kappa^{+}, \forall X \in[\kappa]^{\omega} \exists F \in \mathfrak{C} X \subseteq F .
$$

Remark. (1) is implied by $C H$. The failure of either (2) or (3) is a large cardinal assumption: If the covering lemma holds for $K,(2)$ and (3) will be true. (For (3) it suffices to have an inner model satisfying GCH for which the covering lemma holds. Of course, for $\kappa>2^{\omega}$, (3) just says $\kappa^{\omega}=\kappa^{+}$.)

The proof will be done by inductively constructing an increasing sequence of saturated families on ordinals $\omega \cdot \alpha$. Successor steps can be handled by Condition (1). Limit steps of uncountable cofinality are trivial. For limit ordinals of cofinality $\omega$ that are not cardinals, a construction will be given in $\S 3$. For cardinals of cofinality $\omega$, we will use (2) and (3) from above in $\S 4$.

Thanks to Lajos Soukup for suggesting Condition (3) instead of $\kappa^{\omega}=\kappa^{+}$, and for correcting an error in $\S 4$.

\section{Definitions, Notation, AND A FEW Simple facts}

\subsection{Notation}

$X, X_{1}, \ldots$ : infinite sets.

$x, y, \ldots$ : countable subsets of $X$, i.e. elements of $[X]^{\omega}$.

$\mathfrak{A}, \mathfrak{B}, \ldots$ : almost disjoint families of countable sets, usually $\subseteq[X]^{\omega}$.

$a, a_{1}, \ldots, b, b_{1}, \ldots$ : elements of almost disjoint families.

$Y, Z \ldots$ : subsets of $X$.

2.2. Definition. Let $Y \subseteq X$. An almost disjoint family $\mathfrak{A} \subseteq[X]^{\omega}$ is called saturated with respect to $Y$ if $Y \in I_{\mathfrak{A}}$ or $\exists a \in \mathfrak{A} a \subseteq Y$.

$\mathfrak{A}$ is saturated on $Y$ if $\mathfrak{A}$ is saturated with respect to all $Z \subseteq Y$.

$\mathfrak{A}$ is saturated if $\mathfrak{A}$ is saturated on $X=\bigcup \mathfrak{A}$. (When we talk about a saturated family $\mathfrak{A} \subseteq[X]^{\omega}$, it is understood that $\mathfrak{A}$ is saturated on $X$. But the statement " $\mathfrak{A}$ is saturated on $Y$ " does not by itself imply $\mathfrak{A} \subseteq[Y]^{\omega}$.)

It is easy to see that a saturated family must be a maximal almost disjoint family.

2.3. Fact. If $\mathfrak{A}$ is saturated on $X$, and $\mathfrak{B}$ is saturated on $Y$ ( $X$ and $Y$ almost disjoint), $\mathfrak{C}=\mathfrak{A} \cup \mathfrak{B}$ then $\mathfrak{C}$ is saturated on $X \cup Y$ (assuming $\mathfrak{C}$ is almost disjoint).

2.4. Fact. If $\mathfrak{A}$ is saturated with respect to $Y$, and $\mathfrak{A} \subseteq \mathfrak{B}$, then $\mathfrak{B}$ is saturated with respect to $Y$.

2.5. Definition. For $\mathfrak{A} \subseteq[X]^{\omega}, Y \subseteq X$, let

$$
\mathfrak{A} \mid Y=\{a \cap Y: a \in \mathfrak{A}, a \cap Y \text { infinite }\} .
$$


2.6. Fact. If $\lambda<\kappa$, then $S(\kappa) \rightarrow S(\lambda)$.

Proof. Let $\mathfrak{A} \subseteq[\kappa]^{\omega}$ be an infinite saturated family on $\kappa$. Let $X$ be a subset of $\kappa$ of size $\lambda$ such that $\mathfrak{A} \mid X$ is infinite. (If $\lambda>\omega$, any set of size $\lambda$ will have that property. Otherwise, let $\mathfrak{A}^{\prime} \subseteq \mathfrak{A}$ be countably infinite, and let $X=\bigcup \mathfrak{A}^{\prime}$.) Then $\mathfrak{A} \backslash X$ is an infinite saturated family on $X$.

2.7. Fact. An almost disjoint family $\mathfrak{A} \subseteq[X]^{\omega}$ is saturated iff it is saturated with respect to all countable subsets $y$ of $X$.

Proof. Assume that $\mathfrak{A}$ is saturated with respect to all countable $y \subseteq X$. Let $Y \subseteq X$ be uncountable. Then $Y \in I_{\mathfrak{A}}^{+}$, so we have to find $a \subseteq Y, a \in \mathfrak{A}$. It is clear that $\mathfrak{A}$ is a maximal almost disjoint family. Hence there are infinitely many sets $a_{0}, a_{1}, \ldots$ in $\mathfrak{A}$ that have infinite intersection with $Y$. Let $y=$ $Y \cap \bigcup a_{i} \cdot y$ cannot be almost covered by finitely many sets $b_{1}, \ldots, b_{m} \in \mathfrak{A}$ (otherwise each $a_{i}$ would have infinite intersection with some $b_{k}$ ). Since $\mathfrak{A}$ is saturated with respect to $y, y$ (and hence $Y$ ) contains some $a \in \mathfrak{A}$.

2.8. Fact. If $\mathfrak{A}$ is saturated with respect to $X$, and saturated with respect to $Y$, then $\mathfrak{A}$ is saturated with respect to $X \cup Y$.

2.9. Fact. If $S(\omega)$, then every partition of a countable set into infinitely many countable sets can be extended to a saturated family.

Proof. It is enough to find some saturated family extending some partition of some countable set (into infinitely many countable sets): Take any infinite saturated family $\mathfrak{A}$ on $\omega$, let $a_{0}, a_{1}, \ldots$ be distinct elements of $\mathfrak{A}$. Let $X=\bigcup a_{n}$, $\mathfrak{B}=\mathfrak{A} \uparrow X$. Get $\mathfrak{B}^{\prime}$ by replacing each $a_{i}$ by $a_{i}^{\prime}=a_{i}-\bigcup_{j<i} a_{j}$. Then $\mathfrak{B}^{\prime}$ is saturated (since $I_{\mathfrak{B}}=I_{\mathfrak{B}^{\prime}}$.)

\section{EXTENDING SATURATED FAMILIES}

3.1. Fact. Let $\lambda$ be an ordinal, $c f(\lambda)>\omega$, let $\left(X_{\beta}\right)_{\beta<\lambda}$ be an increasing family of sets, and let $\mathfrak{A}_{0} \subseteq \mathfrak{A}_{1} \subseteq \cdots \subseteq \mathfrak{A}_{\beta} \cdots$ be saturated families on $X_{0}, X_{1}, \ldots, X_{\beta}$, ... respectively (i.e. $\mathfrak{A}_{\beta}$ saturated with respect to all subsets of $X_{\beta}$, but not necessarily $\left.\mathfrak{A}_{\beta} \subseteq\left[X_{\beta}\right]^{\omega}\right)$. Then $\bigcup \mathfrak{A}_{\beta}$ is saturated on $X=\bigcup X_{\beta}$.

Proof. Use 2.7, and note that every countable set $\subseteq X$ is in fact $\subseteq X_{\beta}$, for some $\beta<\lambda$.

The corresponding fact is not true for $c f(\lambda)=\omega$. (Let $\lambda=\bigcup \lambda_{n}, \lambda_{n+1}>\lambda_{n}$ and let $y$ be an infinite set such that $\forall n y \cap \lambda_{n}$ is finite. Then $y$ cannot contain any set in $\bigcup \mathfrak{A}_{\beta}=\bigcup \mathfrak{A}_{\lambda_{n}}$, nor can it be covered by a finite union of such sets.)

However, the following lemma holds:

3.2. Lemma. Let $c f(\lambda)=\omega$, let $\left(X_{\beta}\right)_{\beta<\lambda}$ be an increasing family of sets, and let $\mathfrak{A}_{0} \subseteq \mathfrak{A}_{1} \subseteq \cdots \subseteq \mathfrak{A}_{\beta} \ldots$ be saturated families on $X_{0}, X_{1}, \ldots, X_{\beta}, \ldots$ respectively, and let $\forall \alpha<\lambda \mathfrak{A}_{\alpha} \subseteq \cup_{\beta}\left[X_{\beta}\right]^{\omega}$. Assume there exists a saturated family on $X=\bigcup_{\beta} X_{\beta}$. Then there exists a saturated family $\mathfrak{A}_{\lambda} \supseteq \bigcup_{\beta} \mathfrak{A}_{\beta}$ on $X$. 


\subsection{Corollary}

$$
\begin{aligned}
S(\kappa) & \rightarrow S\left(\kappa^{+}\right), \\
\forall \mu<\lambda S(\mu) & \rightarrow S(\lambda), \quad \text { if } c f(\lambda)>\omega .
\end{aligned}
$$

Proof. Build a chain of families $\mathfrak{A}_{\alpha}$ on $\omega \cdot \alpha$ by induction, starting with a saturated family on $\omega$.

For successor stages, let $\mathfrak{A}_{\alpha+1}=\mathfrak{A}_{\alpha} \cup \mathfrak{B}$, where $\mathfrak{B}$ is any saturated family on $\omega \cdot(\alpha+1)-\omega \cdot \alpha \cdot\left(\mathfrak{A}_{\alpha+1}\right.$ will be saturated by 2.3 .)

If $\alpha$ is a limit ordinal of uncountable cofinality, let

$$
\mathfrak{A}_{\alpha}=\bigcup_{\beta<\alpha} \mathfrak{A}_{\beta} .
$$

(This works, by 3.1.)

If $\alpha$ is a limit ordinal of countable cofinality, let $\mathfrak{A}_{\alpha}$ be a saturated family extending $\bigcup_{\beta<\alpha} \mathfrak{A}_{\beta}$. (Lemma 3.2 applies, since the cardinality of $\alpha$ is $\leq \kappa$ or $<\lambda$, respectively.)

For the proof of 3.2 we may assume w.l.o.g. that $\lambda=\omega$. We may also assume that all $\left(X_{n+1}-X_{n}\right)$ are infinite: If $X_{n_{k+1}}-X_{n_{k}}$ is infinite (for all $k$ ) for some infinite increasing sequence $\left(n_{k}: k \in \omega\right)$, work with the family $\left(X_{n_{k}}: k \in \omega\right)$ instead of $\left(X_{n}: n \in \omega\right)$. Otherwise, there is an $n_{0}$ such that $\forall n \geq n_{0}\left(X_{n+1}-X_{n}\right)$ is finite. Hence for $n \geq n_{0}, \mathfrak{A}_{n}=\mathfrak{A}_{n_{0}}$. If $X-X_{n_{0}}$ is finite, then $\mathfrak{A}_{n_{0}}$ is saturated on $X$. Otherwise, let $\mathfrak{A}_{\omega}=\mathfrak{A}_{n_{0}} \cup \mathfrak{B}$, where $\mathfrak{B}$ is saturated on $X-X_{n_{0}}$. Then $\mathfrak{A}_{\omega}$ is saturated on $X$ and extends all $\mathfrak{A}_{n}$ 's.

Some technology for the proof of 3.2:

3.4. Definition. If $f$ is a function on $\mathfrak{A}\left(\mathfrak{A}\right.$ an almost disjoint family $\left.\subseteq[X]^{\omega}\right)$, and $\forall a \in \mathfrak{A} f(a)$ is an almost disjoint family (not necessarily infinite) $\subseteq[a]^{\omega}$, then let $f_{*}(\mathfrak{A})=\bigcup_{a \in \mathfrak{A}} f(a)$.

3.5. Fact. $f_{*}(\mathfrak{A})$ is almost disjoint. If $\mathfrak{A}$ is saturated on $X$, and if for all $a \in \mathfrak{A}$ $f(a)$ is saturated on $a$, then $f_{*}(\mathfrak{A})$ is saturated.

Proof. It is clear that $f_{*}(\mathfrak{A})$ is almost disjoint.

To show that $f_{*}(\mathfrak{A})$ is saturated, consider any $y \in[X]^{\omega}$. Since $\mathfrak{A}$ is saturated, there are two cases:

Case 1. If $y \supseteq a$, for some $a \in \mathfrak{A}$, then $y \supseteq b$, for any $b \in f(a) .(f(a)$ is not empty, since it is saturated on an infinite set.)

Case 2. If $y \nsupseteq a$, for all $a \in \mathfrak{A}$, then $y \subseteq^{*} a_{1} \cup \cdots \cup a_{n}$, for some sets $a_{1}, \ldots, a_{n} \in \mathfrak{A}$. Let $\mathfrak{B}=f\left(a_{1}\right) \cup \cdots \cup f\left(a_{n}\right)$. Then $\mathfrak{B}$ (and, by 2.4 , also $\left.f_{*}(\mathfrak{A})\right)$ is saturated with respect to $y$, by the following argument:

Let $y^{\prime}=y \cap\left(a_{1} \cup \cdots \cup a_{n}\right)$. Then, by $2.3, \mathfrak{B}$ is saturated with respect to $y^{\prime}$. If $y^{\prime} \supseteq b$ for some $b \in \mathfrak{B}$, then also $y \supseteq b$, and if $y^{\prime} \in I_{\mathfrak{B}}$, then also $y \in I_{\mathfrak{B}}$, since $y$ is almost contained in $y^{\prime}$.

For the following, we fix a family $\left(X_{n}: n \in \omega\right)$ as in the hypothesis of 3.2. 
3.6. Definition. Let $y \in[X]^{\omega}$.

$y$ is called bounded, if $\exists n y \subseteq X_{n}$.

$y$ is called thin, if $\forall n \in \omega y \cap\left(X_{n+1}-X_{n}\right)$ is finite (or $\forall n \in \omega y \cap X_{n}$ is finite).

$y$ is called thick, if $y$ is not bounded, and $\forall n \in \omega y \cap\left(X_{n+1}-X_{n}\right)$ is either infinite or empty.

3.7. Fact. Every $y \in[X]^{\omega}$ is either bounded, thick, thin, or the union of a bounded set and a thin set, or the union of a thick set and a thin set. Hence, by 2.8 ., a family $\mathfrak{A}$ is saturated iff it is saturated with respect to all bounded, thin, and thick sets.

By assumption, $\bigcup \mathfrak{A}_{n}$ contains only bounded sets, and it is clear that $\bigcup \mathfrak{A}_{n}$ (as in the statement of 3.2) is saturated with respect to all bounded sets.

3.8. Fact. Let $\mathfrak{A}$ be a saturated family on $X$. Then there exists a saturated family $\mathfrak{B}$ on $X$ containing only thin, thick and bounded sets.

Proof. For $a \in \mathfrak{A}$, let $f(a)$ be a finite saturated family on $a$, defined as follows: If $a$ is thin, thick or bounded, then let $f(a)=\{a\}$. If $a=b \cup c$, where $b \cap c=\varnothing$, and $b$ and $c$ are thin, thick or bounded, let $f(a)=\{b, c\}$. (By 3.7, this covers all cases.) Now let $\mathfrak{B}=f_{*}(\mathfrak{A})$.

3.9. Lemma. Let $\mathfrak{A}$ be a saturated family on $X$. Then there exists a saturated family $\mathfrak{B}$ containing only thin sets and bounded sets.

Proof. By 3.8, we may assume that all sets in $\mathfrak{A}$ are bounded, thin, or thick. If $a \in \mathfrak{A}$ is bounded or thin, let $f(a)=\{a\}$. Otherwise let $f(a)$ be a saturated family extending the partition on $a$ induced by the representation

$$
a=\bigcup_{n \in \omega}\left(X_{n+1}-X_{n}\right) \cap a \text {. }
$$

Then all sets in $f(a)$ that are not of the form $a \cap\left(X_{n+1}-X_{n}\right)$ are thin. Now let $\mathfrak{B}=f_{*}(\mathfrak{A})$.

3.10. Remark. Clearly, the family $\mathfrak{B}^{\prime}=$ all thin sets in $\mathfrak{B}$ is saturated with respect to all thin subsets of $X$.

3.11. Lemma. Let $\mathfrak{A}$ be an almost disjoint family on $X$ consisting only of thin sets. If $\mathfrak{A}$ is saturated with respect to all thin sets, then $\mathfrak{A}$ is saturated with respect to all thick sets.

Proof. Assume that $y$ is an unbounded thick set that contains no set in $\mathfrak{A}$. This will yield a contradiction.

Construct a sequence $\left(a_{n}: n \in \omega\right)$ of sets from $\mathfrak{A}$ as follows: Given $a_{0}, \ldots$, $a_{n-1}$ let $a_{n} \in \mathfrak{A}$ be a set different from all the $a_{i}(i<n)$, such that $y \cap a_{n}$ is infinite. ( $a_{n}$ exists: Consider the set $y-\bigcup_{j<n} a_{j}$. This set is thick, so it contains some thin set $x$. This set $x$ either contains a set in $\mathfrak{A}$, or is covered by finitely many of them, so in particular it meets some set $a \in \mathfrak{A}$ infinitely 
often, so $\left.a \notin\left\{a_{0}, \ldots, a_{n-1}\right\}\right)$. Let

$$
z=\bigcup_{n}\left(a_{n} \cap y-X_{n}\right) .
$$

Then $z$ is thin:

$$
z \cap X_{k}=\bigcup_{n}\left(a_{n} \cap y-X_{n}\right) \cap X_{k} \subseteq \bigcup_{n<k} a_{n} \cap X_{k}=\text { finite. }
$$

Since $z \subseteq y, z$ contains no member of $\mathfrak{A}$. Therefore it is in $I_{\mathfrak{A}}$ :

$$
z \subseteq \subseteq^{*} b_{1} \cup \cdots \cup b_{m}, b_{k} \in \mathfrak{A} .
$$

Now consider any $a_{n}$ :

$$
a_{n} \cap y-X_{n} \subseteq^{*} b_{1} \cup \cdots \cup b_{m}
$$

Since $a_{n} \cap y-X_{n}$ is infinite, for some $k\left(a_{n} \cap y-X_{n}\right) \cap b_{k}$ must be infinite. But then $a_{n} \cap b_{k}$ is infinite, and since both $a_{n}$ and $b_{k}$ are in the almost disjoint family $\mathfrak{A}, a_{n}=b_{k}$.

This implies that $\left\{a_{0}, a_{1}, \ldots\right\}$, an infinite set, is contained in the finite set $\left\{b_{1}, \ldots, b_{m}\right\}$, a contradiction.

3.12. Lemma. Let $\mathfrak{B}$ be a saturated family on $X$ containing only thin sets and bounded sets. Let $\mathfrak{A}_{0} \subseteq \mathfrak{A}_{1} \subseteq \cdots$ be saturated on $X_{0}, X_{1}, \ldots$, containing only bounded sets. Let

$$
\mathfrak{B}^{\prime}=\{a \in \mathfrak{B}: a \text { is thin }\} .
$$

Then $\mathfrak{A}=\mathfrak{B}^{\prime} \cup \cup \mathfrak{A}_{n}$ is saturated.

Proof. $\mathfrak{A}$ is an almost disjoint family. Clearly $\bigcup \mathfrak{A}_{n}$ is saturated with respect to all bounded sets, and $\mathfrak{B}^{\prime}$ is saturated with respect to thin sets. By $3.11, \mathfrak{B}^{\prime}$ is also saturated with respect to thick sets. By 2.4 we get that $\mathfrak{A}$ is saturated with respect to thin, thick, and bounded sets. But every $y \in[X]^{(1)}$ can be written as a finite union of thin, thick and bounded sets, so by 2.8 we are done.

Proof of 3.2. Let $\mathfrak{A}_{0} \subseteq \mathfrak{A}_{1} \subseteq \cdots$ be saturated on $X_{0}, X_{1}, \ldots$, and assume that $\mathfrak{B}$ is saturated on $X$. By 3.8 and 3.9 there exists a saturated family $\mathfrak{B}_{1}$ containing only thin and bounded sets. By 3.12 we can find a saturated family $\mathfrak{A}=\mathfrak{B}_{1}^{\prime} \cup \cup \mathfrak{A}_{n}$ on $X$ extending $\cup \mathfrak{A}_{n}$.

\section{REACHING CARDINALS OF COUNTABLE COFINALITY}

Lemma. Let $\lambda>\omega, c f(\lambda)=\omega$, and assume $\forall \mu<\lambda S(\mu)$.

Assume that (2) and (3) from $\S 1$ hold. Then $S(\lambda)$.

Proof. Let $\left(C_{\alpha}: \alpha<\lambda^{+}, \alpha\right.$ limit) be a $\square$-sequence, i.e. assume that the following hold for all limit ordinals $\alpha<\lambda^{+}$:

$$
\begin{aligned}
& C_{\alpha} \subseteq \alpha . \\
& C_{\alpha} \text { closed unbounded in } \alpha . \\
& \operatorname{card}\left(C_{\alpha}\right)<\lambda . \\
& \forall \gamma \in C_{\alpha}^{\prime} C_{\gamma}=C_{\alpha} \cap \gamma,
\end{aligned}
$$


where $\operatorname{card}(C)$ is the cardinality of a set $C$, and $C^{\prime}$ is the set of limit points of a set $C$ of ordinals.

Let $\left(\lambda_{n}: n \in \omega\right)$ be an increasing sequence of regular cardinals with limit $\lambda$. Let $k_{\alpha}=\min \left\{k: \operatorname{card}\left(C_{\alpha}\right) \leq \lambda_{k}\right\}$. Let $\left\{x_{\beta}: \beta<\lambda^{+}\right\} \subseteq[\lambda]^{\omega}$ be a covering family, i.e.

$$
\forall x \in[\lambda]^{\omega} \exists \beta: x \subseteq x_{\beta} .
$$

By induction on $\alpha<\lambda^{+}$, we will define

$$
\left(A_{k}^{\alpha}: k \in \omega\right), \quad \mathfrak{A}^{\alpha} \subseteq \bigcup_{k \in \omega}\left[A_{k}^{\alpha}\right]^{\omega}
$$

such that for all $\alpha$ the following will hold:

(a) $A_{k}^{\alpha} \subseteq A_{k+1}^{\alpha}, \quad \operatorname{card}\left(A_{k}^{\alpha}\right)=\lambda_{k}, \quad A_{k}^{0} \subseteq A_{k}^{\alpha}$.

(b) $\forall \beta<\alpha: \exists k^{*} \forall k \geq k^{*} A_{k}^{\beta} \subseteq A_{k}^{\alpha}$.

(c) $\alpha$ limit $\rightarrow \forall \gamma \in C_{\alpha}^{\prime} \forall k \geq k_{\alpha} A_{k}^{\gamma} \subseteq A_{k}^{\alpha}$.

(d) $\mathfrak{A}^{\alpha}$ is an almost disjoint family.

(e) $\mathfrak{A}^{\alpha}$ is saturated with respect to all subsets of $A_{k}^{\alpha}$, for all $k$.

(f) $\forall \beta<\alpha: \mathfrak{A}^{\beta} \subseteq \mathfrak{A}^{\alpha}$.

(g) $\alpha=\beta+1 \rightarrow \exists k x_{\beta} \subseteq A_{k}^{\alpha}$.

We will construct these families by induction on $\alpha$.

Case 1. $\alpha=0$.

Let $A_{k}^{0}=\lambda_{k}$, and let $\mathfrak{A}_{k}^{0} \subseteq\left[\lambda_{k}-\lambda_{k-1}\right]^{\omega}$ be a saturated family on $\lambda_{k}-\lambda_{k-1}$ (which exists by assumption), $\mathfrak{A}^{0}=\bigcup_{k} \mathfrak{A}_{k}^{0}$.

Remark. Since $\lambda=\bigcup_{k} A_{k}^{0}$, (a) implies $\lambda=\bigcup_{k} A_{k}^{\alpha}$ for all $\alpha$.

Case 2. $\alpha$ a limit of uncountable cofinality.

$C_{\alpha}^{\prime}$ must be unbounded in $\alpha$.

Let, for $k<k_{\alpha}, A_{k}^{\alpha}=A_{k}^{0}$.

For any $\gamma_{1}<\gamma_{2}, \gamma_{1}, \gamma_{2} \in C_{\alpha}^{\prime}$ we have $\gamma_{1} \in C_{\gamma_{2}}^{\prime}$. Hence by induction hypothesis (c)

$$
A_{k}^{\gamma_{1}} \subseteq A_{k}^{\gamma_{2}}
$$

for all $k \geq k_{\gamma_{2}}$. (Notice that $k_{\gamma_{2}} \leq k_{\alpha}$, since $C_{\gamma_{2}} \subseteq C_{\alpha}$.)

Let for $k \geq k_{\alpha}$

$$
A_{k}^{\alpha}=\bigcup_{\gamma \in C_{\alpha}^{\prime}} A_{k}^{\gamma},
$$

then $\operatorname{card}\left(A_{k}^{\alpha}\right) \leq \operatorname{card}\left(C_{\alpha}^{\prime}\right) \cdot \lambda_{k} \leq \lambda_{k_{\alpha}} \cdot \lambda_{k}=\lambda_{k}$. Hence (a) holds.

Let

$$
\mathfrak{A}^{\alpha}=\bigcup_{\gamma \in C_{\alpha}^{\prime}} \mathfrak{A}^{\gamma}=\bigcup_{\beta<\alpha} \mathfrak{A}^{\beta} .
$$

By $3.1, \mathfrak{A}^{\alpha}$ is saturated on every $A_{k}^{\alpha}$, so (e) holds. 
(d) follows from the induction hypotheses (d) and (f).

(c) and (f) are true by construction.

(b) follows from (c) and the induction hypothesis.

Case 3. $\alpha$ a limit, $c f(\alpha)=\omega$, and $C_{\alpha}^{\prime}$ is cofinal in $\alpha$. As before, let for $k<k_{\alpha}, A_{k}^{\alpha}=A_{k}^{0}$, and for $k \geq k_{\alpha}$ let

$$
A_{k}^{\alpha}=\bigcup_{\gamma \in C_{\alpha}^{\prime}} A_{k}^{\gamma} \text {, }
$$

then $\operatorname{card}\left(A_{k}^{\alpha}\right) \leq \operatorname{card}\left(C_{\alpha}^{\prime}\right) \cdot \lambda_{k}=\lambda_{k}$. Then (a)-(c) hold.

Let $\gamma_{0}<\gamma_{1}<\cdots$ be an increasing sequence in $C_{\alpha}^{\prime}$, cofinal in $\alpha$. Let $B_{n}=A_{k_{\alpha}+n}^{\gamma_{n}}$. Then

$$
B_{0} \subseteq B_{1} \subseteq \cdots, \quad \lambda=\bigcup_{n<\omega} B_{n}
$$

Let

$$
\mathfrak{A}=\bigcup_{k \in \omega} \mathfrak{A}^{\gamma_{k}}=\bigcup_{\beta<\alpha} \mathfrak{A}^{\beta},
$$

then $\mathfrak{A}$ is saturated with respect to all bounded subsets of $\lambda$, and $\mathfrak{A}$ contains only bounded subsets of $\lambda$. ("bounded", "thin" and "thick" in this paragraph always refers to the representation (*).) There exists a saturated family $\mathfrak{B}_{k} \subseteq$ $\left[A_{k}^{\alpha}-A_{k-1}^{\alpha}\right]^{\omega}$ (since $\operatorname{card}\left(A_{k}^{\alpha}\right)<\lambda$, by (a)). Use 3.9 and 3.10 to find a family $\mathfrak{B}_{k}^{\prime}$ of thin subsets of $A_{k}^{\alpha}-A_{k-1}^{\alpha}$ that is saturated with respect to all thin subsets of $A_{k}^{\alpha}-A_{k-1}^{\alpha}$. Let

$$
\mathfrak{A}^{\alpha}=\mathfrak{A} \cup \bigcup_{k \in \omega} \mathfrak{B}_{k}^{\prime} .
$$

$\mathfrak{A}^{\alpha}$ is almost disjoint (hence (d)), because sets in $\mathfrak{A}$ are bounded and sets in $\mathfrak{B}_{k}^{\prime}$ are thin. As in 3.12 we can use 3.11 to show that $\mathfrak{A}^{\alpha}$ is saturated with respect to all subsets of all $A_{k}^{\alpha}$ 's. This implies (e). (f) is clear.

Case 4. $\alpha$ limit, $C_{\alpha}^{\prime}$ bounded in $\alpha$

Let $\beta_{0}=\sup C_{\alpha}^{\prime}$, and let $\beta_{0}<\beta_{1}<\cdots$ be increasing with limit $\alpha$.

Let $k_{0}=0$, and for $n>0$ let $k_{n}$ be the first number such that

$$
k_{n}>k_{n-1} \text {, }
$$

$$
\forall k \geq k_{n}: A_{k}^{\beta_{n-1}} \subseteq A_{k}^{\beta_{n}} .
$$

(Such a $k_{n}$ exists, by (b)).

Note that this implies:

$$
\forall m \leq n \forall k \geq k_{n}: A_{k}^{\beta_{m}} \subseteq A_{k}^{\beta_{n}} .
$$

Now let, for $k_{n} \leq k<k_{n+1}, A_{k}^{\alpha}=A_{k}^{\beta_{n}}$, and let

$$
\mathfrak{A}^{\alpha}=\bigcup_{\beta<\alpha} \mathfrak{A}^{\beta}=\bigcup_{n \in \omega} \mathfrak{A}^{\beta_{n}} \text {. }
$$


Then we have

$$
\forall k \geq k_{m}: A_{k}^{\beta_{m}} \subseteq A_{k}^{\alpha} \text {. }
$$

Proof. Let $k_{n} \leq k<k_{n+1}$. Then $n \geq m$. Now apply $(* 2)$.

We have to check that (a)-(f) are satisfied for $\alpha$.

Since any $\gamma \in C_{\alpha}^{\prime}$ is $\leq \beta_{0}$, we have for all $k \geq k_{0}(=0), A_{k}^{\gamma} \subseteq A_{k}^{\beta_{0}} \subseteq A_{k}^{\alpha}$. This proves $(\mathrm{c})$.

For any $\beta<\alpha$, let $\beta<\beta_{n}$, then (by $(* 3)$ ) for sufficiently large $k, A_{k}^{\beta} \subseteq$ $A_{k}^{\beta_{n}} \subseteq A_{k}^{\alpha}$. This proves (b).

To show (a), note that $A_{k}^{\alpha} \subseteq A_{k+1}^{\alpha}$ follows easily from the construction if $k+1$ is not of the form $k_{n+1}$. If $k+1=k_{n+1}$, then

$$
A_{k}^{\alpha}=A_{k}^{\beta_{n}} \subseteq A_{k+1}^{\beta_{n}} \subseteq A_{k+1}^{\beta_{n+1}}=A_{k+1}^{\alpha},
$$

where the second inclusion follows from $(* 1)$.

$\mathfrak{A}^{\alpha}$ is saturated on each $A_{k}^{\alpha}$, since $A_{k}^{\alpha}=A_{k}^{\beta}$ for some $\beta<\alpha$, so we can apply the induction hypothesis (e) for $\beta$. Hence (e) holds.

(d) and (f) are clear.

Case 5. $\alpha$ a successor. Let $\alpha=\beta+1$.

If for some $k \quad x_{\beta} \subseteq A_{k}^{\beta}$, then let $A_{k}^{\alpha}=A_{k}^{\beta}$ for all $k$, and $\mathfrak{A}^{\alpha}=\mathfrak{A}^{\beta}$.

Otherwise, let $\mathfrak{B} \subseteq\left[x_{\beta}\right]^{\omega}$ be a saturated family that contains all infinite sets of the form $x_{\beta} \cap\left(A_{k}^{\beta}-A_{k-1}^{\beta}\right)$. Let $\mathfrak{B}^{\prime}$ be the family of all thin sets in $\mathfrak{B}$, with respect to

$$
x_{\beta}=\bigcup_{k}\left(A_{k}^{\beta} \cap x_{\beta}\right) .
$$

Let

$$
\begin{aligned}
\forall k A_{k}^{\alpha} & =A_{k}^{\beta} \cup x_{\beta}, \\
\mathfrak{A}^{\alpha} & =\mathfrak{A}^{\beta} \cup \mathfrak{B}^{\prime} .
\end{aligned}
$$

Then $\mathfrak{A}^{\alpha}$ is an almost disjoint family, and it is saturated with respect to all subsets of $x_{\beta}$.

This finishes the construction of the families $\left(\mathfrak{A}^{\alpha}\right)$. Let $\mathfrak{A}=\bigcup \mathfrak{A}^{\alpha}$. By (f), $\mathfrak{A}$ will be an almost disjoint family. To show that $\mathfrak{A}$ is saturated, consider any $x \in[\lambda]^{\omega}$ : For some $\beta<\lambda^{+}, x \subseteq x_{\beta}$. By the last sentence in Case $5, \mathfrak{A}$ is saturated with respect to $x$.

\section{Conclusion}

$$
V=K \rightarrow \forall \kappa S(\kappa)
$$

and in fact

$$
\text { (1)-(3) from } \S 1 \rightarrow \forall \kappa S(\kappa) \text {. }
$$

Proof. Assume not, and let $\lambda=\min \{\kappa: \neg S(\kappa)\}$. Clearly $\lambda$ is an uncountable cardinal. By the result in $\S 3, \lambda$ cannot be a successor cardinal, and it cannot have uncountable cofinality. By $C H, \lambda>\omega$. Since by definition of $\lambda$ we have $\forall \mu<\lambda S(\mu)$, the construction in this section shows that $S(\lambda)$, a contradiction. 


\section{REFERENCES}

[BDS] B. Balcar, J. Dockalkova, and P. Simon, Almost disjoint families of countable sets, Finite and Infinite Sets, Proc. Coll. Math. Soc. J. Bolyai, I, Eger, 1981.

[EH1] P. Erdös and A. Hajnal, Unsolved problems in set theory, Proc. Sympos. Pure Math. 13 (1971), 17-48.

[EH2] _- Unsolved and solved problems in set theory, Proc. Sympos. Pure Math. 25 (1971), 269-287.

[HJS] A. Hajnal, I. Juhász and L. Soukup, On saturated almost disjoint families, Comment. Math. Univ. Carolina 28 (1987).

[J] T. Jech, Set theory, Academic Press, New York, San Francisco, and London, 1978.

[K] P. Komjath, Dense systems of almost disjoint sets, Finite and Infinite Sets, Proc. Coll. Math. Soc. J. Bolyai, II, Eger, 1981.

(Martin Goldstern, Haim I. Judah) Department of Mathematics, Bar Ilan University, 52900 Ramat GaN, ISRAEL

(Saharon Shelah) Department of Mathematics, Hebrew University of Jerusalem, JERUSALEM, ISRAEL 